\title{
EDGE COLORING NUMBERS OF SOME REGULAR GRAPHS
}

\author{
E. T. PARKER
}

\begin{abstract}
ABSTRACr. The graph composed of $k$ subgraphs, each the totally-disconnected graph with $n$ nodes, arranged in a cycle, can be edge-colored with $2 n$ colors unless $n$ and $k$ are both odd.
\end{abstract}

The edge coloring number of a graph is the smallest number of colors for its edges so that no two adjacent edges have like color. Vizing [1] showed that the edge coloring number is either the maximum degree of a node, or one more than this number.

In this note we consider the edge coloring number of a graph composed of $k$ copies of the totally disconnected graph with $n$ nodes. The totally disconnected graphs are arranged in a $k$-cycle; i.e., two nodes are joined by an edge if and only if they are in components adjacent in the $k$-cycle. We show that the edge coloring number of the graph is $2 n$ unless $n$ and $k$ are both odd.

For $n$ and $k$ both odd, assume that $2 n$ colors suffice to edge-color the graph. Then each node, being of degree $2 n$, has an adjacent edge of each color. Each edge is adjacent to two nodes, thus the edges of a given color must be adjacent to an even number of nodes. The graph has $k n$ nodes, an odd number, giving a contradiction.

For $k$ even a construction of the pattern of colors is given. Designate the nodes in the first totally disconnected component by $A_{0}, \cdots, A_{n-1}$; those in the next component in the cycle by similarly subscripted $B$ 's, etc. Call the colors $X_{0}, \cdots, X_{n-1}$ and $Y_{0}, \cdots, Y_{n-1}$. Color edge $A_{i} B_{j}$ with $X_{i+j}$, the latter subscript reduced $\bmod n$. Color edge $B_{i} C_{j}$ with $Y_{i+j}$. Continue the alternation of $X$ and $Y$ around the cycle of even length $k$.

When $n$ is even the constructive procedure developed below yields an edge coloring. Again, proceeding around the cycle, designate nodes by subscripted $A$ 's, $B$ 's, $\cdots$, etc. Let the colors be $W, X, Y$, and $Z$, each letter assigned $n / 2$ different subscripts. Describe the colorings of the $A_{i} B_{j}$

Received by the editors April 14, 1972.

AMS (MOS) subject classifications (1970). Primary 05 C99.

Key words and phrases. Graph, regular graph, coloring.

(c) American Mathematical Society 1973 
edges by an $n$-by- $n$ array with entries subscripted letters $W, X, Y$, and $Z$, row indices corresponding to $i$ and column indices corresponding to $j$. Each row and each column must have all color entries distinct. The $A B$ coloring array will be compatible with the $B C$ array next in the cycle if column $i$ of the former and row $i$ of the latter contain complementary sets of color letters for each $i$. Partition the nodes of each component $(A, B, \cdots$, etc.) into a first and second half of $n / 2$ nodes each. Color the $A B$ edges according to

$$
\begin{array}{ll}
W & X \\
Y & Z,
\end{array}
$$

where each of the letters means a latin square on the $n / 2$ subscripted letters. For compactness, we write the displayed matrix as $W X Y Z$. Then the $B C, C D$ edges may be colored according to $Z X Y W$ and $W X Y Z$. Also, consecutive sets of edges may be colored by $W X Y Z, Z X W Y$, $Y X Z W, W X Y Z$. A pattern of coloring may be repeated in either two or three steps around the cycle.

\section{REFERENCE}

1. V. G. Vizing, On an estimate of the chromatic class of a p-graph, Diskret. Analiz No. 3 (1964), 25-30. (Russian) MR 31 \#4740.

Department of Mathematics, University of Illinois, Urbana, Illinois 61801 